\title{
El pensamiento crítico interpretativo en Contabilidad y el acto de interpretar las NIIF
}

\author{
Pereira Molina, Gabriel Vicente* \\ *Universidad de Los Andes, Venezuela \\ *Universidad de Los Andes, Programa de Doctorado en Ciencias Contables, Mérida, Venezuela \\ E-mail: gabrielp@ula.ve \\ https://orcid.org/0000-0002-7207-8814
}

Recibido: 20 de noviembre de 2020

Aceptado: 24 diciembre de 2020

\begin{abstract}
Resumen
El presente artículo tiene como objetivo analizar la escuela del pensamiento crítico interpretativo en contabilidad y sus posibilidades para la fundamentación del acto de interpretar las Normas Internacionales de Información Financiera (NIIF). La investigación tiene un carácter cualitativo, se realizó mediante la revisión documental y el análisis de artículos científicos de destacados penadores contables de diversos países. Se concluye que estos postulados científicos de investigación crítico interpretativa, se mantienen neutrales ante los problemas de interpretación de la norma contable conociendo solo la realidad, además que permiten rasgos de subjetividad, lo que no favorece el acto de interpretar el texto normativo (NIIF) de modo objetivo.
\end{abstract}

Palabras clave: Pensamiento crítico interpretativo, interpretación, NIIF, normas contables. 


\title{
Interpretative critical thinking in accounting and the act of interpreting IFRS
}

\begin{abstract}
This article aims to analyze the school of interpretive critical thinking in accounting and its possibilities for the foundation of the act of interpreting the International Financial Reporting Standards (IFRS). The research has a qualitative nature, it was carried out through the documentary review and the analysis of scientific articles by prominent accounting thinkers from various countries. It is concluded that these scientific postulates of interpretive critical research remain neutral in the face of the problems of interpretation of the accounting standard, knowing only the reality, in addition to allowing features of subjectivity, which does not favor the act of interpreting the normative text (IFRS) of objective mode.
\end{abstract}

Keywords: Interpretive critical thinking, Interpretation, IFRS, Accounting Standards.

\section{Introducción}

Para las ciencias contables, un objeto de estudio de interés se halla en las Normas Internacionales de Información Financiera -NIIF o IFRS, estas últimas por sus siglas en inglés- dada su importancia para la práctica profesional en jurisdicciones donde tienen validez; encausadas por el paradigma de la utilidad de la información financiera a la toma de decisiones económicas (Archel, 2007; Farfán, 2010).

Las mencionadas normas requieren sea interpretado el texto normativo para su posterior aplicación, tal y como lo expresa Tagliavia (1995): “...la aplicación requiere interpretación ya que esta pretende descubrir el verdadero sentido...". Debido a que en el acto de interpretar las normas contables, desarrollado por los profesionales de la contabilidad, pueden surgir problemas de interpretación y derivar en imprecisiones, ambigüedades o diversidad de criterios (Casal \& Olaso, 2007); rasgos estos, que deben ser atendidos por un método que permita interpretar las NIIF -validado científicamente-. Esta investigación no pretende dar cuenta de un método interpretativo, solo exponer una óptica de reflexión académica.

En efecto, sobre la base de hechos contables similares se pueden desprender interpretaciones desiguales de las normas contables, acarreando tratamientos contables distintos a eventos económico-financieros semejantes y por ende, información financiera no comparable y no acorde con las necesidades de información financiera útil que exige este modelo contable internacional.

Debido que las NIIF regulan el comportamiento social del quehacer contable y son parte de la interacción humana; surge el interés por desarrollar los principales rasgos teóricos del enfoque interpretativo. En razón de lo anterior, la intencionalidad particular de analizar la escuela del pensamiento crítico interpretativo en contabilidad y sus posibilidades para la fundamentación del acto de interpretar las NIIF.

Por consiguiente, hacer un acercamiento en la búsqueda de una metodología que permita una interpretación uniforme y $\sin$ ambigüedades, facilitando los procesos de obtención de información financiera útil y contribuir a una praxis contable acorde con las necesidades de sus usuarios. En consecuencia, discernir si esta base de pensamiento -la crítico interpretativa- facilita o no un modo idóneo para dicha fundamentación.

Una adecuada fundamentación para interpretar las NIIF, tiene que estar entrelazada con los caracteres o naturaleza de esta norma contable -su esencia 
ontológica y epistemológica-. Desde luego, se debe alcanzar el cumplimiento de las características cualitativas de la información financiera, como también cumplir con las restricciones que el costo de esto puede representar -el beneficio debe ser superior- y cualquier otra condición que exista dentro de este sistema de normas o dentro del Marco Conceptual para la Información Financiera vigente (IFRS Foundation, 2018).

Para efecto de esta investigación, se toma en cuenta la característica cualitativa de mejora: Comparabilidad (IFRS Foundation, 2018); ya que producto de la interpretación de la norma contable y de la materialización de la actividad contable, se produce información financiera que busca satisfacer el parámetro comparativo, entre otros aspectos. El acto interpretativo de la norma contribuye en este aspecto.

La comparabilidad se relaciona con la congruencia que existe en la información financiera y permite identificar diferencias o semejanzas entre partidas, en adelante sería cotejable la información financiera a lo largo de la vida económica de la organización, tomando en consideración rasgos que evalúan financieramente a la entidad en el tiempo y contrastable con entidades similares (IFRS Foundation, 2018). Existe una asociación directa de esta característica con la interpretación de las NIIF, a pesar que no necesariamente la comparabilidad debe materializarse en información financiera uniforme - de una sola forma o método aplicar-. Las circunstancias de la realidad del evento económico repercuten en la información construida contablemente.

Sobre este sentido, el órgano emisor de las NIIF menciona en su Marco Conceptual: "la información sobre una entidad es más útil si puede ser comparada con información similar sobre otras entidades, así como con información similar sobre la misma entidad para otro periodo u otra fecha" (IFRS Foundation, 2018, p. 17); es decir, bajo la premisa de que una entidad posea características similares a otra, hace posible comparar su información financiera y da el valor de hacerse más útil a la misma, en correspondencia también con los enunciados cualitativos fundamentales de este sistema normativo -relevancia, representación fiel, entre otros-; todo esto mejora el proceso de toma de decisiones económicas en la empresa y el acto interpretativo de las NIIF incide en este fin.

Ahora bien, en otro orden de ideas, la perspectiva que se tenga de la realidad en relación a las NIIF con respecto a su interpretación, representa un elemento significativo. Se deben considerar las diversas posibilidades al momento que un intérpreteinvestigador quiera abordar esta actividad, por lo tanto, hay que tener en cuenta que en líneas generales pueden ser: El plano idealista, en esencia concibe que la realidad se construye a partir del pensamiento humano y el intercambio social; esto, hace posible un conocimiento amplio y coherente dependiendo de su aceptación, no obstante, otra perspectiva puede ser, el plano realista, cuyo planteamiento es que la realidad es independiente del sujeto, buscando solo que el sujeto la conozca, estudie, represente o interprete objetivamente (Carrizo \& León, 2007).

Ambos criterios ontológicos mencionados -el idealista y el realista- de amplia discusión a lo largo de la historia, no escapa del debate en contabilidad, aunado a la esencia epistemológica y metodológica que puede influir en investigaciones que se materialicen sobre este tema particular, donde el abordaje empírico o racional definiría el desarrollo de cualquier aporte teórico. En este caso, trascendiendo del horizonte del pensamiento dominante -positivista- a otra visión de la investigación científica de tipo interpretativo.

Por consiguiente, partiendo de los principales rasgos teóricos del pensamiento crítico-interpretativo en contabilidad y de una aproximación al acto de interpretación de la norma contable que realizan los contadores profesionales, se plantea la siguiente interrogante ¿Cuáles son las posibilidades para que sobre la base del pensamiento crítico interpretativo en contabilidad sea desarrollado un método que permita interpretar el texto de las NIIF?

A continuación, en la investigación, se presenta su abordaje metodológico haciendo la distinción de su carácter cualitativo. Luego se desarrollan las 
premisas del pensamiento crítico interpretativo en contabilidad en conjunto con una aproximación al acto de interpretar las NIIF, posteriormente se analizan otros detalles de esta base de pensamiento que muestra el paradigma de la contabilidad como una práctica social e institucional en su contexto interpretativo y por último se presenta una conclusión.

\section{Metodología}

Para el desarrollo de la presente investigación, se toman en cuenta parámetros de orden cualitativo, esto conlleva la revisión documental y el análisis de contenido de artículos científicos y libros relacionados desde una perspectiva semántica y lógica, mediante lo cual, se identifican los principales rasgos de la corriente crítico interpretativa y se denotan algunos aspectos del acto de interpretar las NIIF.

Macías (2013) señala: “...los estudios interpretativos son radicalmente subjetivistas..." (p. 704). Visto de esta forma, se expresan ideas de la contabilidad, que muestran rasgos de subjetividad, propio de esta postura alternativa de investigación contable. De este modo, servir de sustento o refutación en el desarrollo teórico del acto de interpretar las NIIF o para la construcción de un método de interpretación mediante esta postura.

Lo anterior implica que puedan existir vicios de sesgo. Pero, son mitigados mediante: Un objetivo claro y preciso, un método preplaneado que permite referenciar autores que investigan estos aspectos teóricos e inferir lógica y descriptivamente esta base de pensamiento en contabilidad sobre información relevante en el área, cuya producción científica se ha materializado por investigadores destacados en revistas científicas y arbitradas conforme a las exigencias internacionales y que han proporcionado resultados firmes (Macías, 2013), aceptados por la comunidad científica contable alineada a este enfoque. Se muestran los aspectos más resaltantes que describen destacados pensadores contables de diversos países.

3. Premisas del pensamiento crítico-interpretativo en contabilidad para la interpretación de las NIIF

El pensamiento crítico interpretativo contable, representa una corriente epistemológica alternativa a la dominante -positivista-, para el estudio, profundización y construcción de conocimiento en el mundo de la contabilidad. Anthony Hopwood (1944-2010), ha sido considerado uno de sus precursores y principales líderes intelectuales; esta propuesta desarrolla un compendio reflexivo que concibe el conocimiento contable con postulados diferenciados del positivismo y el pensamiento crítico social; en tanto, permite conocer la realidad contable desde múltiples matices para desarrollar conocimiento científico (Agudelo, 2013; Aquel \& Cicerchia, 2011; Irausquín, 2012; Macías, 2013; Paredes, 2007; Villarreal \& Córdoba, 2017).

Es importante acotar, que no debemos confundir dicha escuela del pensamiento como eje epistemológico, con el acto de interpretar la norma contable -NIIF-. Esto último, "interpretar" una norma, contempla el acto de averiguar el apropiado sentido del texto (Casal \& Olaso, 2007), esclareciendo la intencionalidad dada en este y a su vez la guía correcta que marcará la pauta a la actividad contable.

Este enfoque investigativo tiene como propósito que los investigadores indaguen en el fenómeno de interés, para comprender los procesos de interacción del hombre en la sociedad -en este caso los contadores profesionales intérpretes de la norma-, de tal modo, luego de descifrar el contenido de la actividad contable mediante el discurso generado, se busca tener claridad de la magnitud del pensamiento humano y sus amplias posibilidades constructivas, atribuyendo significado a esos eventos que realizan los sujetos (Gómez, 2007; Larrinaga, 1999; Irausquín, 2012).

De acuerdo con lo anterior, se señala que la investigación interpretativa en contabilidad subyace en referencia que: "la realidad social es una construcción humana y que, por lo tanto, sólo podemos comprender un hecho social acudiendo e interpretando a sus actores" (Larrinaga, 1999, p.114). Esto fundamenta que los investigadores

Pereira Molina, Gabriel Vicente

El pensamiento crítico interpretativo en Contabilidad y el acto de interpretar las NIIF 
contables apegados a éstos principios; conocen y comprenden una realidad particular a través de la interpretación de los hechos que se producen por la interacción social de los profesionales contables y no mediante la solución de problemas de la contabilidad -este último elemento se debe tener en cuenta, en el caso que se pretenda dar solución a las fallas interpretando normas contables-.

Es posible que se manifiesten elementos de subjetividad al momento de interpretar la norma; expresado mediante las palabras o en definitiva plasmado en la contabilidad - o información financiera de la entidad-, validado en algunos casos por el juicio del profesional de la contabilidad. Por consiguiente, la investigación interpretativa da cabida a entender y comprender los detalles asumidos por el profesional contable para conocer profundamente una realidad que puede resultar muy compleja - interpretar la norma-.

Por esto, el pensamiento interpretativo contable se caracteriza por ser amplio y hasta en la necesidad de apoyarse en diversas fuentes teóricas -contables, económicas, financieras, jurídicas, lógicas, entre otras- para tener una mejor aproximación al conocimiento de un fenómeno, justificado por el hecho que el profesional contable se involucra con estas teorías desde su formación y es el responsable de aplicarlas en su conjunto, en este fin, siempre afrontaría la realidad del hecho contable en función de su criterio y considerando su interacción con la comunidad contable (elementos subjetivos). Cabe resaltar, que esto debería hacerse sin menoscabo de lo que establecen los principios o normas contables, si se busca una interpretación objetiva, precisa y clara de las NIIF.

Obviamente, las relaciones humanas dan cabida a la profesión contable en sus máximas de formación y experiencia en el campo laboral, tácitamente, esto implica un discurso reflexivo de los presupuestos contables -normas y teorías-, dando sustento a su actuación profesional con base a las exigencias que pueden existir en algunas jurisdicciones -mediante leyes, costumbres, entre otras-, haciendo coercitivo o no el cumplimiento de dichos presupuestos teóricos o normativos de la contabilidad.
Por lo tanto, los interesados en indagar sobre esa realidad en contabilidad -la interpretación de las NIIF-, desde esta perspectiva, tienen que adentrarse en el panorama de ideas de la interacción discursiva de la sociedad de contadores, para poder descifrar y comprender las posturas asumidas con respecto de algún evento económico y su tratamiento en contabilidad. En correspondencia con esto, se dice: “...Las prácticas contables están inmersas en una realidad dinámica, en la cual las representaciones de las cosas dependen de las particularidades que experimentan las personas involucradas en ellas..." (Macías, 2013, pp. 703-704). Esto facilitaría un proceso de comprensión de la intersubjetividad contable, pero repercutiría en la aplicación uniforme de los planteamientos normativos, especialmente en su modo objetivo.

De tal modo, se infiere que la realidad del acto de interpretar se circunscribe en esta perspectiva al entorno social -la sociedad que rodea al sujeto intérprete- y la investigación interpretativa intervendría en el descubrimiento y comprensión de cualquier fenómeno -como los problemas de interpretación que puedan presentarse-, consecuentemente se captarían los elementos contables y no contables de esta actividad exegética a través del discernimiento que hacen los sujetos mediante el lenguaje -conceptualizaciones, juicios y razonamientos-, configurando la interpretación discursiva, es decir, valorar los discursos hablados y escritos (Archel, 2007; Larrinaga, 1999; Macías, 2013; Puxty, 1993; Ramis, 2006).

Para lo anterior, se involucran aspectos cognoscitivos del profesional de la contabilidad con el conjunto de significados sociales existentes -relacionados con cultura, costumbres, sistemas normativos, sistemas de poder, entre otros- que predominan evidentemente en él y son identificados, definidos y aceptados por la sociedad contable (Quintero, 2019; Puxty, 1993).

El investigador que se alinea al eje epistemológico crítico-interpretativo, presupone que existe cierto significado construido socialmente, sobre el que puede surgir alguna comprensión -conocimiento, interpretación o reflexión-. A su vez, los seres 
humanos -contadores profesionales- actuarían de acuerdo a los significados que las cosas tienen para ellos y ese significado será el resultado de la interacción social, lo más importante es, que esos significados requieren que el individuo permanezca activo en el proceso de interpretación para justificar su entendimiento y por ende, implica desentrañar el lenguaje y su validez (Quintero, 2019; Larrinaga, 1999; Puxty, 1993; Ramis, 2006).

En estas ideas, la actuación del contador profesional se perfecciona en función del significado que la sociedad contable fija sobre las cosas organización en general, entes reguladores, entre otros- y dicho profesional lo acepta, haciéndolo parte de su dinámica de trabajo. Por lo tanto, la investigación interpretativa conocería esa realidad intangible pero posible -problemas de interpretación de las NIIF- y desde esos caracteres mencionados surgirían los aportes teóricos que contribuyen a la ciencia contable.

En ideas de la profesora australiana Chua (1986) sobre planteamientos de la investigación interpretativa, refleja que la teoría adecuada a su esencia lógica, se usa para explicar las intenciones humanas; cada sujeto producto de su aprehensión cognoscitiva desde los inicios de su formación contable, va asumiendo discrecionalmente de cada teoría o lineamiento normativo su propia percepción, sin menoscabar los mecanismos lógicos de dichas bases teóricas.

Mediante estas perspectivas se facilita explicar las intenciones y acciones del contador profesional interpretando las NIIF. Se añade también, la participación de la comunidad de profesionales contables y demás miembros involucrados con la contabilidad, asumiendo posturas que pueden llegar en algún momento a permear en la teoría o práctica de la contabilidad, interviniendo la escuela interpretativa en explorar éstas particularidades.

En relación con lo expuesto, Chua (1986) plantea que la realidad en estos parámetros, se articula y configura a partir de actividad social y es objetiva mediante este mismo mecanismo. Entonces, desde la ocurrencia del hecho contable, el proceso de interpretación de la norma hasta el producto de la contabilidad -información financiera-, va inmersa la actuación humana y la singularidad intencional que se refleja en la práctica de la contabilidad mediante la implementación de cualquier acto uso de técnicas o métodos contables, criterios de presentación de información financiera, entre otros-; siendo objetivado esto, si desde un punto de vista social se considera de ese modo.

Esto último surge, por los consensos teóricos de los sujetos contables que se desenvuelven en la sociedad y los referentes sobre temas contables -teorías o desarrollo doctrinario de aceptación generalizadafijados en función de patrones históricos $\mathrm{y} / \mathrm{o}$ lingüísticos aceptados. Complementado, además, por la aplicación obligatoria de las normas de orden técnico.

Ahora bien, Chua (1986) distingue la vinculación directa de la teoría con la práctica, la primera, tratando de explicar la segunda, respectivamente. Es fundamental evaluar la conducta del profesional contable, mediante un análisis de los postulados teóricos contables -donde se deben incluir aspectos normativos-, ya que no sería coherente hacer argumentaciones sobre prácticas en contabilidad, en jurisdicciones o ambientes donde determinaciones teóricas o normativas no tengan las mismas delimitaciones sociales, esto solo haría posible el desarrollo de factores comparativos de la práctica contable.

Sin lugar a duda, esta escuela del pensamiento tiene la disposición de ampliar la construcción de conocimiento contable sobre una base que se distingue del pensamiento dominante. En el caso interpretativo, se sustentaría en una realidad construida por los sujetos y abordando el profesional de la ciencia contable una investigación neutral -no influye en la realidad, solo la conoce-. Es así, que se dice que la investigación interpretativa: “... está desprovista de prejuicios y de intenciones de intervención...” (Macías, 2013, p. 704).

Se debe mencionar la salvedad, que los enfoques de tipo hermenéutico estrechamente ligados a área sociales, no llegan a abordar la realidad como los 
enfoques crítico-sociales, los cuales parten de algunos supuestos asociados a las necesidades humanas, perdiendo neutralidad (Larrinaga, 1999).

El análisis precedente vislumbra, cómo otras disciplinas pueden apoyar con bases teóricas a las ciencias contables en la construcción de nuevo conocimiento sobre estas premisas interpretativas, así: La sociología, las ciencias políticas, el derecho, entre otras; hacen que la contabilidad se nutra del entorno y logre una mejor comprensión de los fenómenos, para fortalecer su desarrollo científico de tipo hermenéutico. Esto permite estudiar aspectos abstractos y entender la actividad humana y/o contable, mediante: La psicología, la cultura, entre otras áreas del conocimiento, facilitando elementos de comprensión del complejo mundo económicofinanciero que se asocia a la interpretación normativa (Archel, 2007; Aquel \& Cicerchia, 2011; Vargas, 2013).

En relación a esto, proponen algunos elementos metodológicos, que facilitan el descubrimiento de hallazgos que pueden impulsar la investigación crítico-interpretativa y se dice:

[...] Para abordar el estudio de la contabilidad, así entendida, la corriente interpretativa propone al lenguaje $\mathrm{y}$ la conversación como instrumento de investigación y traducirá a través de entrevistas semiestructuradas, observación participante, revisión de documentos, estudios históricos o estudios de casos [...] (Aquel \& Cicerchia, 2011, p. 22)

En lo anterior, se refleja el papel que tienen algunas técnicas de recolección de información, adoptadas al momento de captar datos de un fenómeno y profundizar en su entendimiento.

Dependiendo del objeto de estudio, se marca la pauta sobre el alcance de dichas técnicas. Esto contribuye en descifrar el lenguaje inmerso dentro de la actividad interpretativa, procesado mediante los actos cognitivos de la mente pero que se perfeccionan en la comunicación, mediante el lenguaje verbal o escrito.
Por consiguiente, en contabilidad se dispone desde la etapa contable del registro -reconocimiento, en ambiente NIIF- o antes, hasta la elaboración de los estados financieros -preparación de información financiera, en ambiente NIIF- y toma de decisiones económicas, en su esencia de querer interpretar el lenguaje construido socialmente en la actividad contable, acarreando valoraciones subjetivas y distanciándose del carácter objetivo de las NIIF.

Sobre las valoraciones subjetivas del pensamiento interpretativo, se toma en consideración argumentos históricos, sociales, lingüísticos y sistemáticos aplicados en la contabilidad para lograr una mejor comprensión del entorno (Archel, 2007; Gómez, 2005; Macías, 2013), pero se corre un riesgo al interpretar la norma, al cambiar alguno de esos factores o sus circunstancias, va a variar el resultado de lo interpretado.

La realidad que trae consigo la interpretación de las NIIF, sobre la cual pueden recaer variables cualitativas como: Intereses económicos, sistemas de poder, tiempo... influyen en el acto de interpretar y de aplicar determinada política contable, pero se debe perseverar en interpretar las NIIF, en su carácter racional y lógico, tomando en consideración los constructos sobre que sustentan dicho sistema normativo.

Mediante este sistema normativo la contabilidad necesariamente va a redibujar -intentar representar del modo más idéntico posible- un evento económico, el cual, para ser admitido dentro de un proceso de contabilización, debe tomar en cuenta indicaciones expresas en las etapas de reconocimiento, medición, presentación, revelación y/o de baja en cuentas del evento económico (IFRS Foundation, 2018), pero el carácter utilitario a la toma de decisiones económicas, prescinde de la neutralidad que el pensamiento interpretativo amerita.

\section{Otras perspectivas del pensamiento crítico interpretativo contable $y$ sus posibilidades para la interpretación de la norma contable}

Una apreciación sobre la que se puede acotar, es 
que se vincula a la contabilidad como una práctica social e institucional, donde se toma en cuenta a la actividad contable con un grado mayor de complejidad que la simple técnica contable, esta tendría una influencia no solo para la organización, sino también para la sociedad en general. Convirtiéndose en un paradigma investigativo a nivel a mundial, asociado con todos los elementos científicos de la contabilidad como una ciencia social (Larrinaga, 1999; Potter, 2005).

Desde la vista de este paradigma de investigación, Miller (1994) considera algunos elementos que distinguen esta forma de ver la contabilidad como: Técnica, lógicas de la contabilidad y dominio contable. Desde un punto de vista de la contabilidad como técnica, el investigador crítico interpretativo participa sobre el desarrollo de la práctica contable y tiene presente los hechos que sean de su interés y capten la atención al manifestarse lo intersubjetivo. En sí, esto permite comprender hasta cómo los grupos de poder influyen en la práctica contable, cómo los profesionales contables deciden aplicar determinado método o política en contabilidad y otros rasgos que se proyecten a través de la técnica en contabilidad.

Evidentemente, considerar la contabilidad como técnica, puede aportar elementos de la realidad en diversos contextos para que sean estudiados y así explorar consecuencias de estas, sean intencionales o no (Potter, 2005). Simplificado mediante la observación o análisis para encontrar hallazgos que den origen o continuidad a reflexiones, cuestionamientos y/o referencias de interés por conocer, tanto en la organización como en la sociedad en general.

Con relación a las lógicas de la contabilidad, un factor fundamental de la contabilidad está en su lenguaje especializado - extensamente desarrollado en las NIIF- y en los significados que la contabilidad adopta para poder construir su sustento teórico y práctico. Los constructos lógicos contables, permiten correlacionar armónicamente los elementos de la contabilidad con toda su complejidad. De este modo se toma en consideración cualidades que incluyen conceptos, características, mediciones, entre otros.
El elemento cognoscitivo que requiere el pensamiento interpretativo contable va de la mano en favorecer los procesos lógicos del mensaje contable, para entender con precisión, razones y fundamentos de los órganos reguladores desarrollando las normas, como también, todo aquello que se asocie al contador profesional en el desempeño de su actividad y la trascendencia o intercambio dentro de su entorno.

La última distinción de la contabilidad como práctica social e institucional, está en función del dominio de la contabilidad, ejerciendo influencia sobre eventos económicos y sus matices financieros (Miller, 1994; Potter, 2005). Donde una amplia dinámica de factores convergen en la praxis contable presentando muchos aspectos de interés que contribuyen a que la contabilidad deba rediseñarse para incorporar aspectos que deben ser tomados en cuenta.

A medida que se expande el desarrollo científico contable y se producen implementaciones de aceptación general, repercute en actos de la actividad profesional y en tanto sea manifestado, los investigadores podrán adherirse a esa realidad contable para aportar a la contabilidad como ciencia, en estas premisas que se han expresado.

Cualquier evento susceptible de ser contabilizado, con alguna trascendencia en la realidad y de interés para las ciencias contables, en forma de cuantificaciones, revelaciones, cualificaciones u otra variable que pretenda hacer valer estas perspectivas, sean nuevas - diseñadas; rediseñadas- marca la pauta en las teorizaciones motivadas por el dominio de la práctica contable (Potter, 2005). El desempeño contable, puede hacer que se proyecten aportes interesantes, que maximizan los recursos dentro y fuera de la organización.

De acuerdo a lo mencionado, destaca el auge en los últimos años de la literatura contable (Potter, 2005) - en muchos aspectos-, entre los cuales se encuentra; la regulación contable internacional ligada al pensamiento dominante, pero permite romper la línea imaginaria de la frontera de los países en cuanto a contabilidad se refiere, 
para dar apertura a los discursos de contadores profesionales que se desempañan en ambientes académicos - investigativos y de cualquier otro interesado en contribuir teóricamente a las ciencias contables. Apoyado sobre la base de estas premisas, contribuiría considerablemente al debate científico interpretativo.

El inexorable aporte lingüístico que se desprende de lo anterior, favorece el aprovechamiento del pensamiento crítico interpretativo y sus métodos, para contribuir con las organizaciones, su crecimiento financiero, el orden cultural, indagando hasta en las bases de la sociedad - valores y principios-. Conociendo posibles aciertos o desaciertos en los cuales la contabilidad participa.

No solo el positivismo puede contribuir en la expansión de las ciencias contables, sino que la hermenéutica puede desempeñar una función significativa en este papel, en referencia de lo cual, se dice: "La posibilidad del pensamiento contable admite y exige la construcción de conocimiento contable desde diversas posturas epistemológicas, concepciones ontológicas y alternativas metodológicas." (Machado, 2012, p. 232).

Se amplía la visión investigativa, para no solo tener la óptica del ente material del fenómeno contable -factores objetivos e independientes del sujeto-, sino ver también la posibilidad de aprender del sujeto y su actuación -factores subjetivos que pueden ser objetivados-. Mediante el hecho de interpretar normas se vinculan caracteres mentales del sujeto y de impulso simbólico expresado a través de lo verbal y la escritura.

La naturaleza humana conlleva que el ser, en su conducta social e individual, pueda trascender por condiciones que nutren cualquier acontecimiento. Derivado de la capacidad que puede llegar a tener la mente humana: Describiendo, evaluando, analizando, infiriendo e interpretando, para luego representar en lenguaje un hecho cualquiera, del cual no escapa tampoco el hecho contable - o la actividad contable-. Esto se refleja en los estudios interpretativos, dando valor a la interacción entre sujetos y su capacidad constructiva.
Ahora bien, en relación al acto de interpretar las NIIF y su aproximación para consolidar las bases teóricas apropiadas de un posible método de interpretación -aceptado científicamente-, involucra de manera directa un fin utilitario a la práctica profesional, donde los rasgos subjetivos y el entorno no deben fijar las premisas interpretativas -contribuiría en aumentar las posibilidades o formas interpretativas-, distanciándose de la esencia objetivista de la norma contable, afectando su interpretación precisa.

El enfoque interpretativo estaría en favor de comprender las múltiples posibilidades subjetivas al momento de interpretar la norma y no de homogeneizar parte de esta actividad contable - de orden funcionalista-. En cuanto a nuevos aportes teóricos interpretativos hay que tener presente

[...]En la pretensión de construir pensamiento contable, la Contabilidad debe referir la realidad, las organizaciones, la circulación de valor y comprenderlos en sus diversas expresiones; ello implica abandonar la tradicional autoreferencia a prácticas y actividades contables, así como a criterios para elaborar estados financieros cuando se desarrollan los conceptos de teoría, modelo y sistema [...] (Machado, 2012, p. 234)

En este sentido, se debe tomar en cuenta que la compleja realidad de la contabilidad en relación a interpretar las NIIF, la investigación interpretativa podría facilitar hallazgos $\mathrm{o}$ mecanismos interpretativos, dando paso a descifrar cualquier expresión de los sujetos intérpretes. Pero debe quedar claro, que las NIIF en su desarrollo, plantean elementos prácticos -el paradigma de la utilidad- que deben ser atendidos por los profesionales con intención de evitar múltiples formas de interpretación, que pueden distanciarse de esta esencia, su distinción lógica, ontológica y epistemológica.

El profesional contable, debe materializar en contabilidad efectos financieros y económicos, apegados a lineamientos normativos definidos por estructuras lógicas y racionales que buscan seguridad jurídica (Molina Sánchez \& Tua Pereda, 
2010) para representar activos, pasivos, patrimonio, ingresos, costos y gastos desde un punto de vista cuantitativo y cualitativo. La estructura financiera de la organización debe expresar objetivamente la realidad; en cambio desde horizontes interpretativos se puede reflexionar sobre estos actos contables y sus repercusiones para la organización y la sociedad. El desempeño del profesional de la contabilidad interpretando la norma, hace que se proyecten oportunidades interesantes en estas perspectivas, pero no para participar activamente y fijar bases de interpretación al texto de las NIIF.

\section{Conclusiones}

Para finalizar, dentro de la diversidad de posibilidades para la construcción de conocimiento en las ciencias contables, la investigación crítico interpretativa se presenta como una alternativa que favorece aspectos subjetivos, facilitando el discernimiento y comprensión de cualquier hecho que se vincule a la actividad de la contabilidad y al acto de interpretación de las NIIF, destacando la importancia del entorno y su influencia en eventos que la hermenéutica va a buscar entender y del mismo modo, darlos a conocer a la sociedad de contadores, sin cuestionar, si es favorable o no al interés organizacional de tomar decisiones económicas, tal y como lo prevén las NIIF. Es decir, la investigación interpretativa se mantiene neutral, no participa activamente para intentar corregir cualquier falla de interpretación de la norma contable.

Por lo tanto, el acto de interpretar las NIIF puede verse desfavorecido en criterios objetivos. La información financiera que se produzca sobre la base de este sistema normativo, debe seguir el paradigma de la utilidad de la información por parte de sus usuarios. En tanto, la interpretación y aplicación de la norma debe considerarse en parámetros donde la subjetividad, no contribuya a desarrollar múltiples criterios de interpretación, que impulsan el hecho de que dicha información no se apegue a las características cualitativas establecidas en el marco conceptual de la IFRS Foundation (2018), en especial, la comparabilidad. Por consecuencia, se puede ver afectada la actividad contable en su fin del paradigma de la utilidad.

\section{Referencias bibliográficas}

Agudelo Vargas, M.V. (2013). Análisis de los aportes de Anthony Hopwood a la disciplina contable. Lúmina, (14), 290-315.

Aquel, S. \& Cicerchia, L. (2011). Alternativas vigentes en investigación contable: una introducción a su estudio. SaberEs: Revista de Ciencias Económicas y Estadística, Universidad Nacional de Rosario, (3), 15-26.

Archel, P. (2007). Discurso contable, ideología e informes anuales: un enfoque interpretativo. Contaduría Universidad de Antioquia, (51), 4164.

Carrizo, W., \& León, S. (2007). ¿Qué realidad representa la contabilidad? Pecvnia: Revista de la Facultad de Ciencias Económicas y Empresariales, Universidad de León, 0(5), 1727. doi:http://dx.doi.org/10.18002/pec.v0i5.709

Casal, J.M. \& Olaso, L.M. (2007). Curso de introducción al derecho: Introducción a la Teoría General del Derecho. Caracas: Publicaciones Universidad Católica Andrés Bello.

Chua, W.F. (1986). Radical Developments in Accounting Thought. The Accounting Review, 51(4), 601-632.

Farfán L, M.A. (2010). Análisis de la vigencia del paradigma de utilidad en la contabilidad moderna. Lúmina, (11), 242-263.

Gómez, M. (2005). Breve introducción al estado del arte de la orientación crítica en la disciplina contable. Contaduría Universidad de Antioquia, (43), 113-132.

Gómez Villegas, M. (2007). Comentarios sobre el aprendizaje-construcción de la teoría contable. Lúmina, 07, 129-153. https://doi.org/10.30554/ lumina.07.1180.2006

IFRS Foundation. (2018). Marco Conceptual para la Información Financiera. Londres: Consejo de Normas Internacionales de Contabilidad.

Irausquín, C.A. (2012). Perspectivas hermenéuticas Pereira Molina, Gabriel Vicente 
como metodología en la investigación de las ciencias contables. Multiciencias, 12(2), 167173.

Larrinaga, C. (1999). Perspectivas alternativas de investigación en contabilidad: Una revisión. Revista de contabilidad. Spanish accounting review, 02(03), 103-131.

Machado, M. (2012). Del positivismo a la diversidad epistemológica: un paso necesario para la construcción de pensamiento contable. Contaduría Universidad de Antioquia, (60), 219-237.

Macías, H. (2013). Vínculos de la investigación contable interpretativa con la producción académica colombiana: avances y oportunidades, Cuadernos de Contabilidad, 14(35), 699-727.

Miller, P. (1994). Accounting as a social and institutional practice: an introduction. En A. Hopwood and P. Miller (Eds.), Accounting as a social and institutional practice (pp. 1-36). Cambridge University Press.

Molina Sánchez, H. y Tua Pereda, J. (2010). Reglas versus Principios contables, ¿son modelos incompatibles? Revista Española de Financiación y Contabilidad, 39(146), 259-287.

Paredes, G. (2007). Críticas Epistemológicas y Metodológicas a la Concepción Positivista en las Ciencias Sociales, ACADEMIA, 6(12), 2442.

Potter, B.N. (2005). Accounting as a Social and Institucional Practice: Perspectives to Enrich our Understanding of Accounting Change. Abacus, 41(3), 265-289.

Puxty, A.G. (1993). The Social \& Organizational Context of Management Accounting. Advanced Management Accounting and Finance Series. CIMA. San Diego: Academic Press.

Quintero Cardona, A.C. (2019). Teoría, práctica y praxis en contabilidad: una aproximación desde el materialism dialéctico. Lúmina, (20), 102-120. https://doi.org/10.30554/ lumina.20.2856.2019
Ramis, P. (2006). Lógica y crítica del discurso. Mérida: Consejo de Publicaciones Universidad de Los Andes.

Tagliavia, A. (1995). LA INTERPRETACIÓN DESDE LA TEORÍA DEL DERECHO. Tesis de doctorado no publicada, Universidad Complutense de Madrid, Madrid.

Vargas Restrepo, C. M. (2013). Caracterización de la investigacion contable. El aporte de Chua. Lúmina, (14), 262-289. https://doi. org/10.30554/lumina.14.1082.2013

Villarreal, J., \& Córdoba Martínez, J. (2017). Fundamentos para el estudio de las corrientes de investigación en contabilidad. Tendencias, 18(2), 139-151. https://doi.org/10.22267/ rtend.171802.81 